\title{
A study on timelike sweeping surfaces and singularities in Minkowski 3-Space $\{\mathrm{E}\}_{-}\{1\}^{\wedge}\{3\}$
}

\author{
Fatemah Mofarreh ${ }^{1}$, Rashad Abdel-Baky², and Nadia alluhaibi ${ }^{3}$ \\ ${ }^{1}$ Princess Nourah Bint Abdulrahman University Central University \\ ${ }^{2}$ Assiut University \\ ${ }^{3}$ King Abdulaziz University
}

July 19, 2020

\begin{abstract}
The main aim of this paper is the use of the rotation minimizing frame for the singularity type classification of timelike sweeping surface in Minkowski 3-Space $\$ \backslash \operatorname{mathbb}\{\mathrm{E}\}_{-}\{1\}^{\wedge}\{3\} \$$. Then, we give conditions for a timelike sweeping surface to be developable ruled surfaces. To apply and illustrate the main results, some examples are given.\%
\end{abstract}

\section{Hosted file}

A study on timelike.pdf available at https://authorea.com/users/305115/articles/470719-astudy-on-timelike-sweeping-surfaces-and-singularities-in-minkowski-3-space-e-_-1-3 


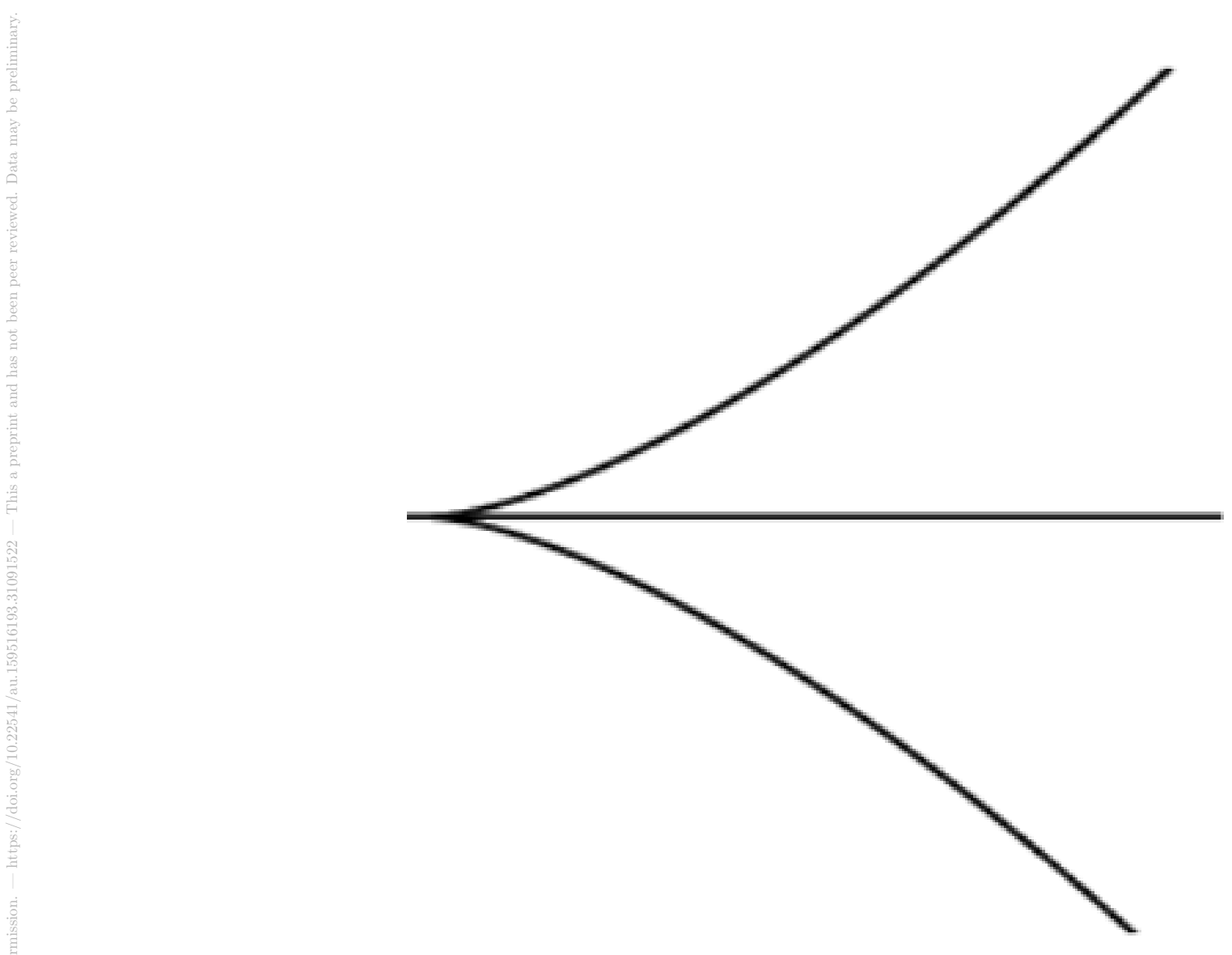

2 


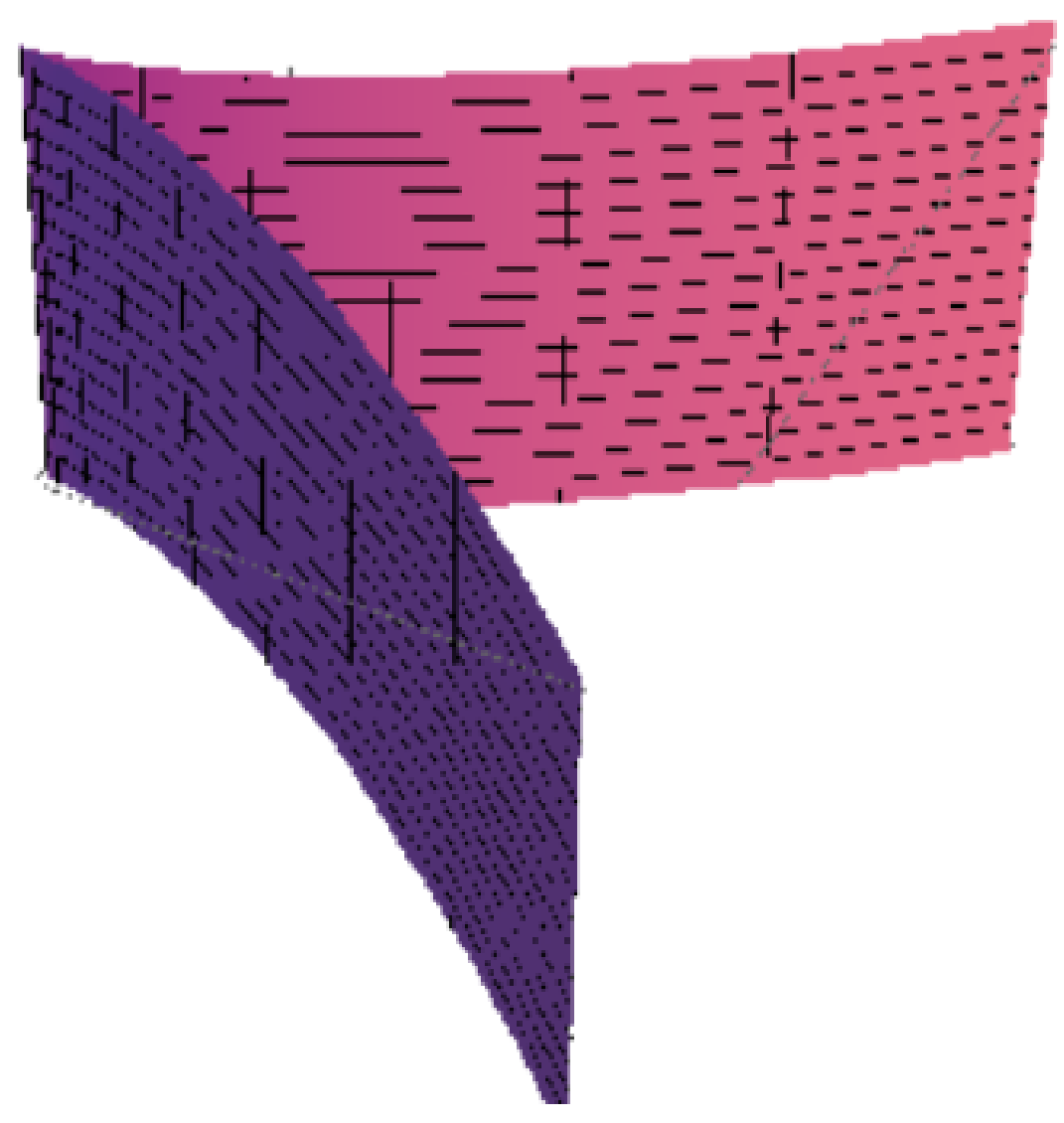




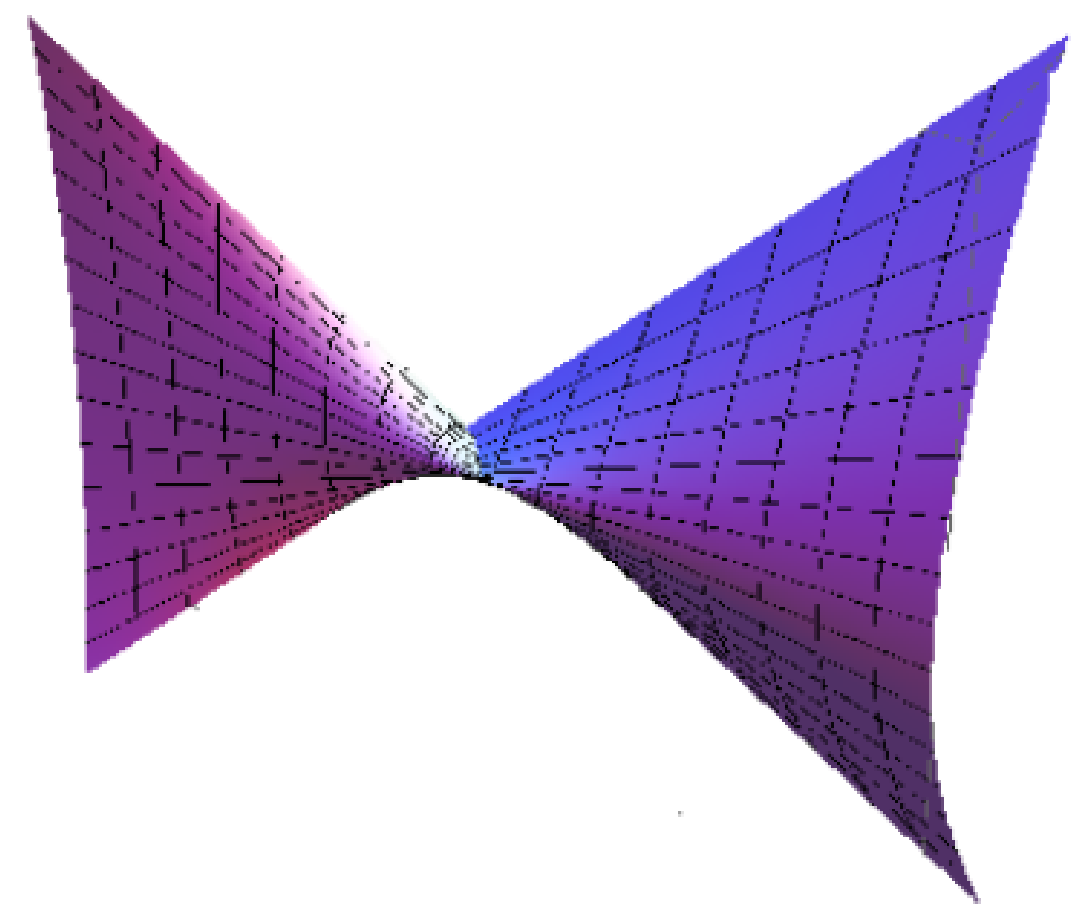




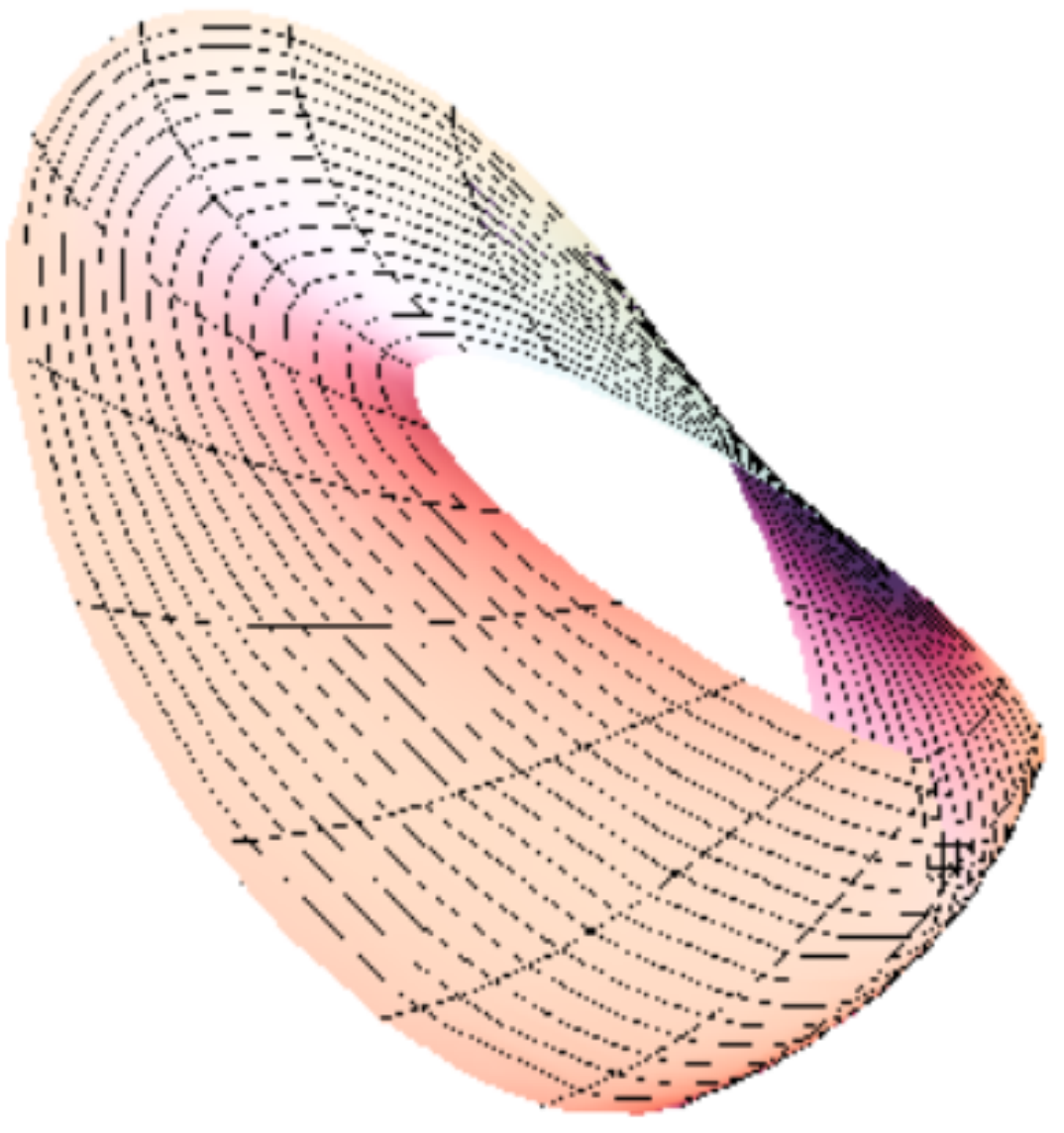




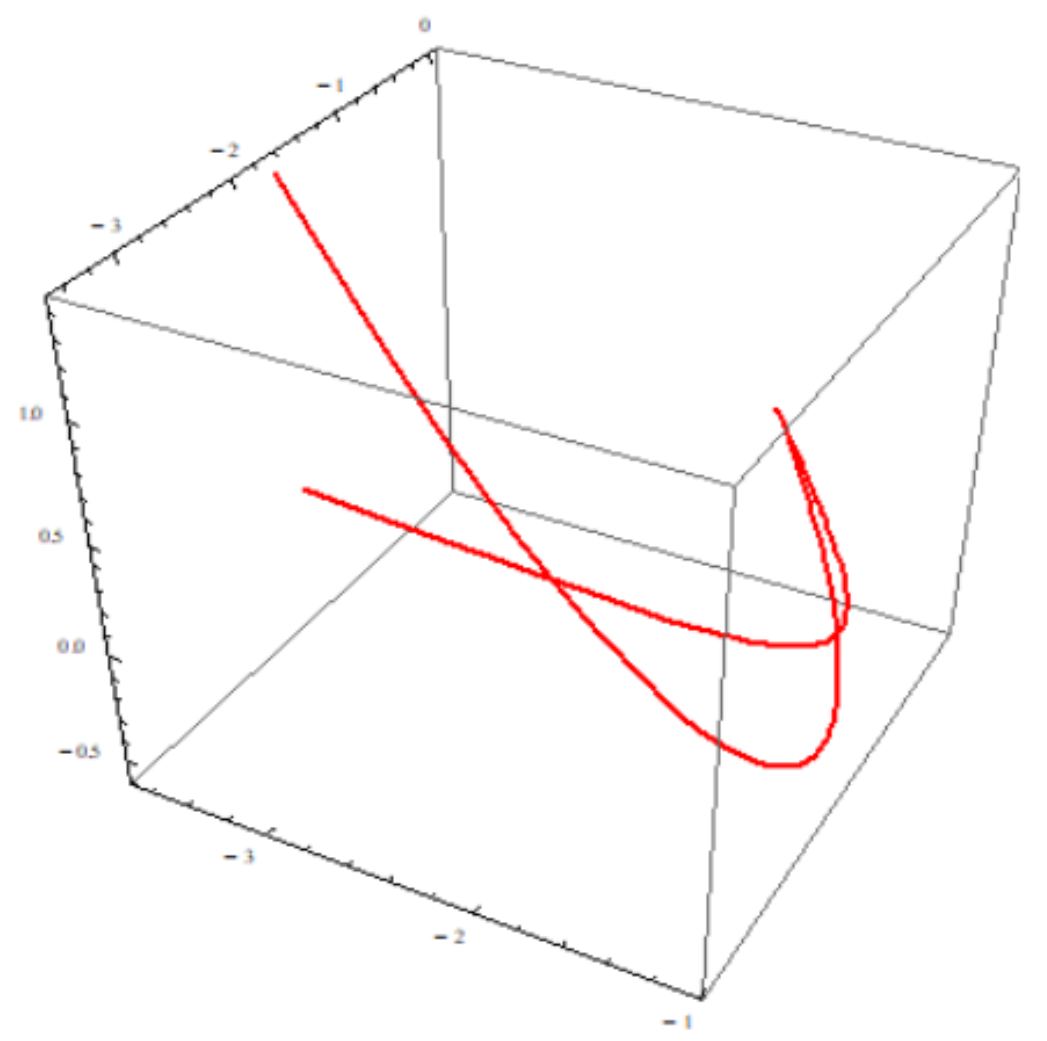

